\title{
COPD is associated with reduced pulmonary interstitial expression of pentraxin-3
}

\author{
G.R. Van Pottelberge*, K.R. Bracke*, N.S. Pauwels*, F.E. Vermassen\#, \\ G.F. Joos* and G.G. Brusselle*
}

ABSTRACT: Pentraxin (PTX)3 is involved in antimicrobial defence, apoptotic cell clearance and extracellular matrix stability. As these processes are altered in chronic obstructive pulmonary disease (COPD), we aimed to investigate PTX3 expression in patients with this disease.

PTX3 expression was quantified by immunohistochemical staining of lung tissue from neversmokers, smokers without COPD, and in patients with COPD of Global Initiative for Chronic Obstructive Lung Disease (GOLD) stage I, II and III-IV. mRNA expression was examined in total lung tissue by quantitative RT-PCR. PTX3 concentration was measured in induced sputum and plasma by ELISA.

PTX3 is mainly localised in the interstitium of the small airways and alveolar walls. There were no significant differences in pulmonary, sputum and plasma PTX3 expression between study groups. However, PTX3 expression in small airways correlated significantly with forced expiratory volume in $1 \mathrm{~s}(\mathrm{r}=\mathbf{0 . 3 5}, \mathrm{p}=\mathbf{0 . 0 0 4})$. In the alveolar walls, PTX3 expression correlated significantly with carbon monoxide transfer coefficient $(r=0.28, p=0.04)$. In sputum, PTX3 levels were highly correlated with the number of neutrophils. Finally, systemic levels of PTX3 tended to be lower in severe COPD compared with mild COPD.

In COPD, airflow limitation and reduced transfer coefficient for carbon monoxide are associated with lower pulmonary interstitial expression of PTX3.

KEYWORDS: Airway inflammation, chronic obstructive pulmonary disease, extracellular matrix, induced sputum, innate immunity

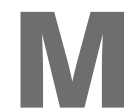
orbidity and mortality due to chronic obstructive pulmonary disease (COPD) are increasing worldwide [1]. The World Health Organization predicts that COPD will be listed as the fourth most important cause of death by 2030 [2]. The accelerated decline in lung function in patients with COPD is usually progressive and, until now, no medical treatment has proved to slow down this process.

COPD is generally considered as an inflammatory lung disease, in which inhaled noxious particles and fumes trigger an activation of the innate and adaptive immune response [3]. This inflammatory response contributes to the four main mechanisms altering the small airways and alveoli structurally and functionally: oxidative stress, proteolysis, apoptosis and remodelling. These mechanisms result in the two main pathological features of COPD: chronic bronchiolitis (with a narrowing of the small airways) and emphysema (destruction of the alveolar walls) $[4,5]$.

The factors that drive the ongoing inflammation in COPD remain to be elucidated. Impaired immune defence mechanisms leading to lowgrade viral infections and bacterial colonisation in the small airways of patients with COPD could contribute to this process [6-8]. In addition, reduced clearance of apoptotic cells and autoimmune mechanisms could also play a role in maintaining destructive inflammation in the lungs of patients with COPD [9-12].

Pentraxin (PTX)3 is a member of the long PTXs, which are humoral factors of the innate immune response. This molecule is produced by different
AFFILIATIONS

*Laboratory for Translational Research in Obstructive Pulmonary Diseases, Dept of Respiratory

Medicine, and

\#Dept of Thoracic and Vascular Surgery, Ghent University Hospital, Ghent, Belgium.

CORRESPONDENCE

G.R. Van Pottelberge Dept of Respiratory Medicine Ghent University Hospital $7 \mathrm{~K} 12 \mathrm{IE}$ De Pintelaan 185 B9000 Ghent Belgium E-mail: geert.vanpottelberge@ ugent.be

Received:

Aug 272010

Accepted after revision: Aug 092011

First published online:

Sept 152011 
cell types (white blood cells, fibroblasts, endothelial cells, epithelial cells, chondrocytes, synoviocytes and smooth muscle cells). Tumour necrosis factor (TNF)- $\alpha$ and interleukin- $1 \beta$ are main inducers of PTX3 expression [13-15]. PTX3 is stored in specific granules in neutrophils from which it is swiftly released in response to microbial recognition and inflammatory signals. Released PTX3 is partially found in neutrophil extracellular traps, which are formed by extruded DNA [16].

Similar to C-reactive protein (CRP; which is a short PTX), PTX3 is capable of opsonisation of microbes. In addition, PTX3 is involved in clearance of apoptotic cells. Besides the innate defence functions, PTX3 is also involved in the formation and stability of the extracellular matrix. PTX3 is, through binding with TNF- $\alpha$-stimulated gene 6 (TSG-6), an anchorage point for hyaluronic acid cross-linking, which is a main component of the extracellular matrix $[17,18]$.

Serum concentrations of PTX3 rapidly increase during inflammatory conditions, such as sepsis and acute myocardial infarction $[19,20]$. Increased PTX3 expression is also present in severe chronic inflammatory conditions, such as rheumatoid arthritis [21]. In contrast, low-grade systemic inflammation, as detected, for instance, in metabolic syndrome, is not accompanied by increased systemic PTX3 levels [22]. The role of PTX3 in pulmonary infection and acute lung injury is reviewed elsewhere [23].

In summary, PTX3 has several important functions that could be relevant to COPD pathogenesis. We hypothesise that PTX3 expression is altered in COPD, contributing to the increased susceptibility to low-grade infections and possible autoimmune responses in COPD. Apart from its immunological role, altered PTX3 expression with reduced hyaluronic acid cross-linking could interfere with structural processes, such as remodelling and emphysema.

\section{MATERIALS AND METHODS}

A detailed description of the materials and methods used is available in the online supplementary material. All patients signed written informed consent prior to participation in the study. This study was approved by the Medical Ethical Committees of Ghent University Hospital (Ghent, Belgium) and University Hospital Gasthuisberg (Leuven, Belgium) in accordance with the Declaration of Helsinki.

\section{Lung tissue}

Lung tissue was obtained from surgical lung resection specimens of patients diagnosed with solitary pulmonary tumours at Ghent University Hospital and from lung explants of endstage COPD patients undergoing lung transplantation at University Hospital Gasthuisberg.

Paraffin-embedded lung tissue sections were stained with antihuman PTX3 antibody (clone MNB4; Alexis Corporation, Lausen, Switzerland). Airways free of cartilage and adjacent pulmonary arteries were analysed with a computerised image analysis system (KS400; Zeiss, Oberkochen, Germany). Expression of PTX3 in the alveoli was determined using a semiquantitative score by two independent observers, blinded for clinical data.
Expression of target genes (PTX3 and TNF- $\alpha$ ) and reference genes in lung tissue mRNA was analysed with TaqMan Gene Expression Assays (Applied Biosystems, Foster City, CA, USA). RT-PCR was performed in duplicate using diluted cDNA template and the LightCycler480 Probes Master Mix (Roche, Basel, Switzerland).

\section{Sputum induction and processing}

Sputum induction and processing was performed as described previously [24]. Samples were analysed by ELISA for human PTX3 (Alexis Corporation).

\section{Plasma PTX3 and CRP levels}

Samples were analysed by ELISA for human PTX3 (Alexis Corporation) and high-sensitivity ELISA for CRP (R\&D Systems, Abingdon, UK).

\section{Statistical analysis}

Statistical analyses were performed using SPSS software (SPSS Inc., Chicago, IL, USA). Differences between multiple study groups were evaluated by the Kruskal-Wallis test, followed by group-to-group comparison using the Mann-Whitney U-test.

Correlations between clinical data and log-transformed image analysis data were obtained using the Pearson correlation coefficient. Linear regression was used to investigate the association between image analysis data and forced expiratory volume in $1 \mathrm{~s}$ (FEV1). A p-value of $<0.05$ was considered significant.

\section{RESULTS}

\section{Protein expression of PTX3 in human lungs}

Figure 1 shows representative slides of immunohistochemical staining of PTX3 in human lung tissue. PTX3 was expressed in the small airways, mainly with an extracellular localisation in the lamina propria and adventitia (fig. 1a). A similar pattern of PTX3 expression was identified in adjacent pulmonary blood vessels. Figure $1 \mathrm{~b}$ shows the appropriate isotype control staining. Figure 1c shows expression of PTX3 in the alveolar compartment. PTX3 was present in type II pneumocytes. Figure 1d shows expression of PTX3 in the alveolar macrophages.

\section{Quantification of PTX3 in lungs of never-smokers, smokers without COPD and patients with COPD}

Quantification of PTX3 protein expression in small airways

The characteristics of the study population are provided in table 1 . The population consisted of 66 patients: never-smokers $(n=10)$, smokers without COPD $(n=16)$, and patients with COPD in Global Initiative for Chronic Obstructive Lung Disease (GOLD) stages I ( $n=13)$, II $(n=14)$ and III-IV $(n=13)$.

Representative sections of human lung tissue stained for PTX3 in smokers without COPD and patients with COPD are shown in figure $1 e$ and $f$, respectively. Using computerised image analysis, the level of expression of PTX3 was measured in the walls of the small airways. In severe and very severe COPD, the level of PTX3 in the small airway wall tended to be lower compared with mild COPD and subjects without COPD. There were no significant differences in PTX3 expression between study groups (fig. 2a). However, the expression of PTX3 (area of positive staining as a percentage of the total airway wall) 

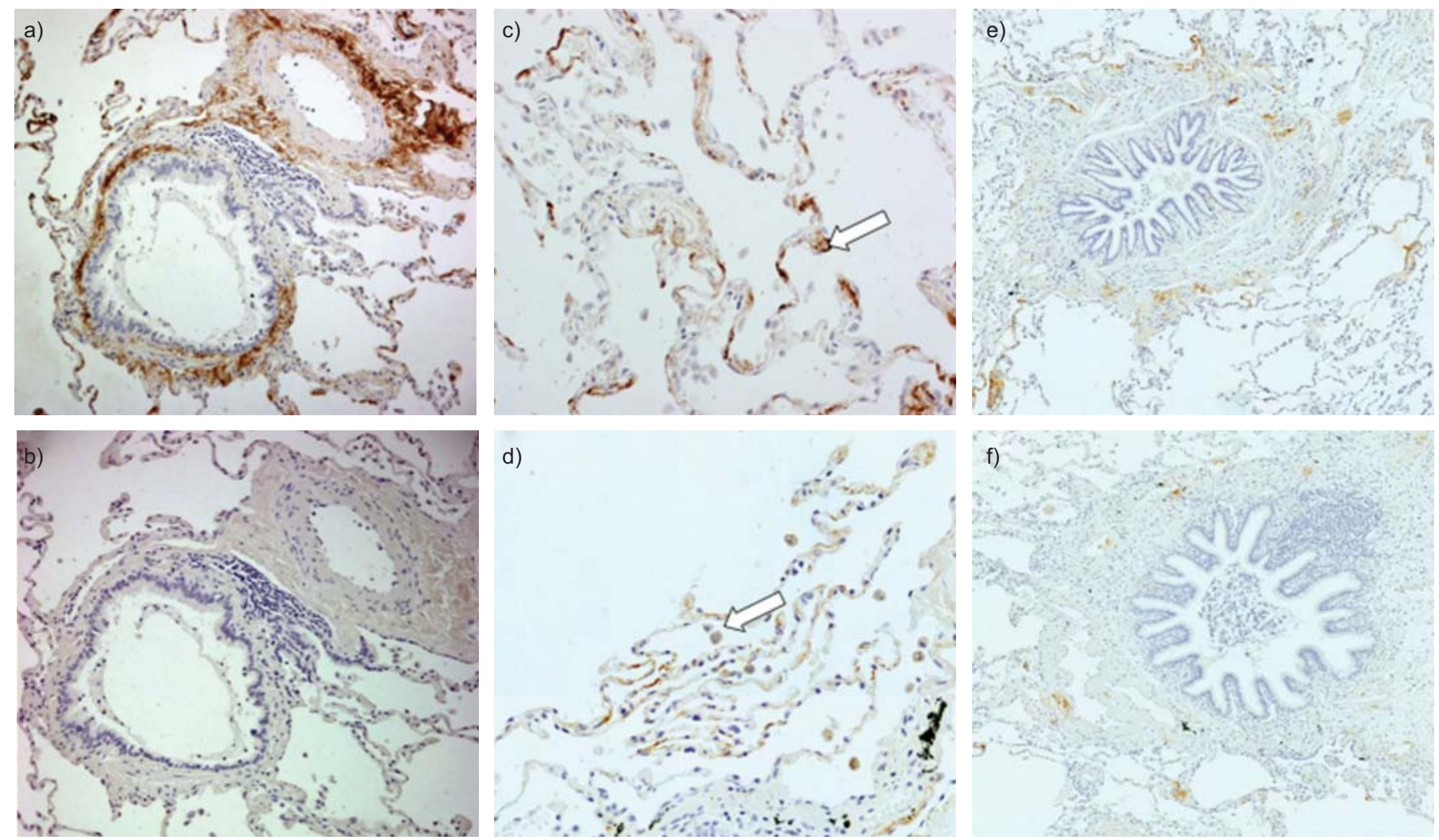

FIGURE 1. Immunohistochemical staining for pentraxin (PTX)3 in human lungs. Paraffin-embedded sections were incubated with anti-human PTX3 antibody. PTX 3 expression is visualised by brown 3,3'-diaminobenzidine staining. Expression of PTX3 was located in the interstitium of the lamina propria and adventitia of the small airways and in the walls of pulmonary arteries. Alveolar septa, alveolar macrophages and type II pneumocytes also contained PTX3. a) Anti-human PTX3 antibody staining in small airway and pulmonary artery. b) Isotype control staining. c) Staining for PTX3 in the alveolar compartment. Arrow: type II pneumocyte. d) PTX3 expression in the alveoli. Arrow: alveolar macrophage. Sections are from a never-smoker without airway obstruction. e) Representative slide of a smoker without chronic obstructive pulmonary disease (COPD). f) Representative slide of a patient with COPD in Global Initiative for Chronic Obstructive Lung Disease stage III.

correlated significantly with the post-bronchodilator FEV1 \% predicted (Pearson correlation coefficient $\mathrm{r}=0.35, \mathrm{p}=0.004$ ) (fig. 2b) and with the FEV1/forced vital capacity ratio $(r=0.27$, $\mathrm{p}=0.031$ ) (data not shown). When normalising the area of positive staining for the length of the basement membrane, the correlation between FEV1 and PTX3 expression remained significant $(\mathrm{r}=0.31, \mathrm{p}=0.012)$. There were no significant differences in PTX3 expression between current smokers and ex-smokers (data not shown). Univariate analysis of PTX3 expression in relation to clinical and biochemical variables is shown in table 2. PTX3 expression correlated significantly with parameters of airway obstruction, but not with other biological variables.

The effect of possible confounders on the expression of PTX3 in small airways was investigated by linear regression model (table 3). The association between PTX3 expression levels and FEV1 remained significant after adjustment for variables that changed the point estimate of FEV1 by $>10 \%$ (treatment with inhaled corticosteroids, treatment with oral corticosteroids and time since quit smoking).

Quantification of PTX3 protein expression in pulmonary arteries Expression of PTX3 in pulmonary arteries, located adjacent to the small airways, was measured using image analysis. PTX3 expression in the pulmonary artery wall was not different between study groups (fig. E-1 in the online supplementary material). The level of PTX3 expression correlated significantly with the transfer coefficient of the lung for carbon monoxide (KCO) $(r=0.30, p=0.039)$ (fig. $E-2$ in the online supplementary material).

\section{Quantification of PTX3 protein expression in alveoli}

The mean alveolar expression of PTX3, assessed by a semiquantitative score, was not different between study groups (fig. 3a). However, alveolar PTX3 levels correlated significantly with $\mathrm{KCO}$, as shown in figure $3 \mathrm{~b}(\mathrm{r}=0.28, \mathrm{p}=0.040)$. Univariate analysis is shown in E-table 1 in the online supplementary material.

\section{Quantification of PTX3 expression at mRNA level in lung tissue}

The characteristics of the study population are shown in Etable 2 in the online supplementary material. The mRNA expression of PTX3 was not different between study groups. There was no correlation between the expression of PTX3 and FEV1 \% pred ( $\mathrm{r}=-0.09, \mathrm{p}=0.52)$ (data not shown) or between the alveolar score of PTX3 and KCO $(r=0.19, \mathrm{p}=0.27)$ (data not shown). PTX3 mRNA expression levels in total lung significantly correlated with the mRNA expression of TNF- $\alpha(r=0.325, p=0.024)$ (fig. 4a). mRNA expression data 
TABLE 1 Characteristics of the study population for the immunohistochemical study ${ }^{\#}$

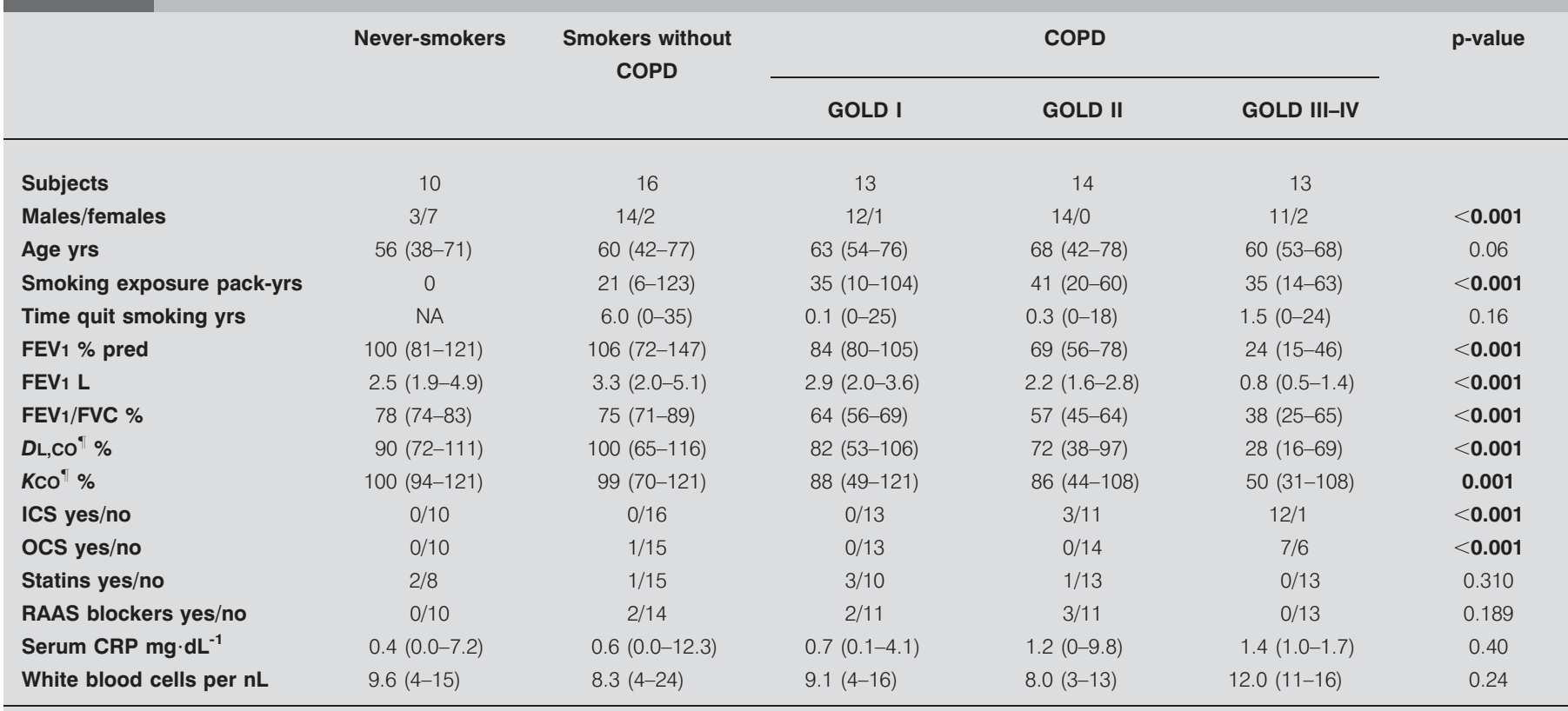

Data are expressed as n or median (range), unless otherwise stated. COPD: chronic obstructive pulmonary disease; GOLD: Global Initiative for Chronic Obstructive Lung Disease; FEV1: forced expiratory volume in $1 \mathrm{~s}$; \% pred: \% predicted; FVC: forced vital capacity; DL,CO: diffusing capacity of the lung for carbon monoxide; Kco: transfer coefficient of the lung for carbon monoxide; ICS: inhaled corticosteroids; OCS: oral corticosteroids; RAAS: renin/angiotensin/aldosterone system; CRP: C-reactive protein; NA: not applicable. Bold indicates statistically significant result by Kruskal-Wallis test for continuous variables and Fisher's exact for categorical variables. $\#: n=66 ;{ }^{\#}: 14$ missing values.

for TNF- $\alpha$ are shown in figure E-3 in the online supplementary material.

\section{Expression of PTX3 in induced sputum}

Characteristics of the study population are shown in E-table 3 in the online supplementary material and the results of the PTX3 ELISA in induced sputum supernatant are shown in Etable 5 in the online supplementary material. There were no significant differences between study groups. There were no correlations between FEV1 and the concentration of PTX3 in induced sputum supernatant (data not shown). PTX3 levels correlated significantly with the number of neutrophils in induced sputum $(\mathrm{r}=0.66, \mathrm{p}<0.001)$ (fig. $4 \mathrm{~b})$.

\section{PTX3 expression in plasma}

The characteristics of the study population are shown in Etable 4 in the online supplementary material. In contrast to high-sensitivity CRP levels in plasma, the concentrations of plasma PTX3 were not different between study groups (Etable 5 in the online supplementary material). Plasma PTX3 concentrations did not correlate with FEV1 $(\mathrm{r}=0.08, \mathrm{p}=0.56)$ (fig. E-4 in the online supplementary material). However, in the subgroup of patients with COPD, the level of circulating PTX3 was significantly correlated with FEV1 $(r=0.37, \mathrm{p}=0.04)$. In contrast, plasma CRP levels correlated significantly with FEV1 $(r=-0.32, p=0.014)$ (data not shown).

\section{DISCUSSION}

To our knowledge, this is the first study addressing the expression of PTX3 in human lung tissue sections. Importantly, this study revealed a marked association between airflow limitation, reduced $\mathrm{KCO}$ and pulmonary expression of PTX3 in COPD.

This study demonstrated the expression of PTX3, both at the mRNA and protein levels, in human lung tissue. PTX3 expression was observed in pulmonary white blood cells and type II pneumocytes, which is compatible with previous in vivo studies in mice and in human cells in vitro [25-28]. Apart from the presence of PTX3 in inflammatory cells and alveolar epithelial cells, we also observed an important extracellular location of PTX3, especially in the subepithelial regions and adventitia of the small airways, in the alveolar walls, and in the walls of pulmonary arteries. The pattern of interstitial localisation of PTX3 has previously been reported in myocardium and kidney $[29,30]$.

The mean protein expression levels of PTX3 in airways walls were not significantly different between study groups. However, there was a clear trend towards lower expression of PTX3 in small airways of patients with COPD compared with subjects without airflow limitation. Indeed, there was a significant correlation between FEV1 (a marker for small airway obstruction) and the expression of PTX3 in the small airway wall. This marked independent association of FEV1 and PTX3 expression in the small airways links the low levels of PTX3 to the most important feature of COPD: airway obstruction. This association remained significant, even when adjusting for possible confounders.

In contrast, there was no significant difference between study groups regarding the level of PTX3 expression in pulmonary 

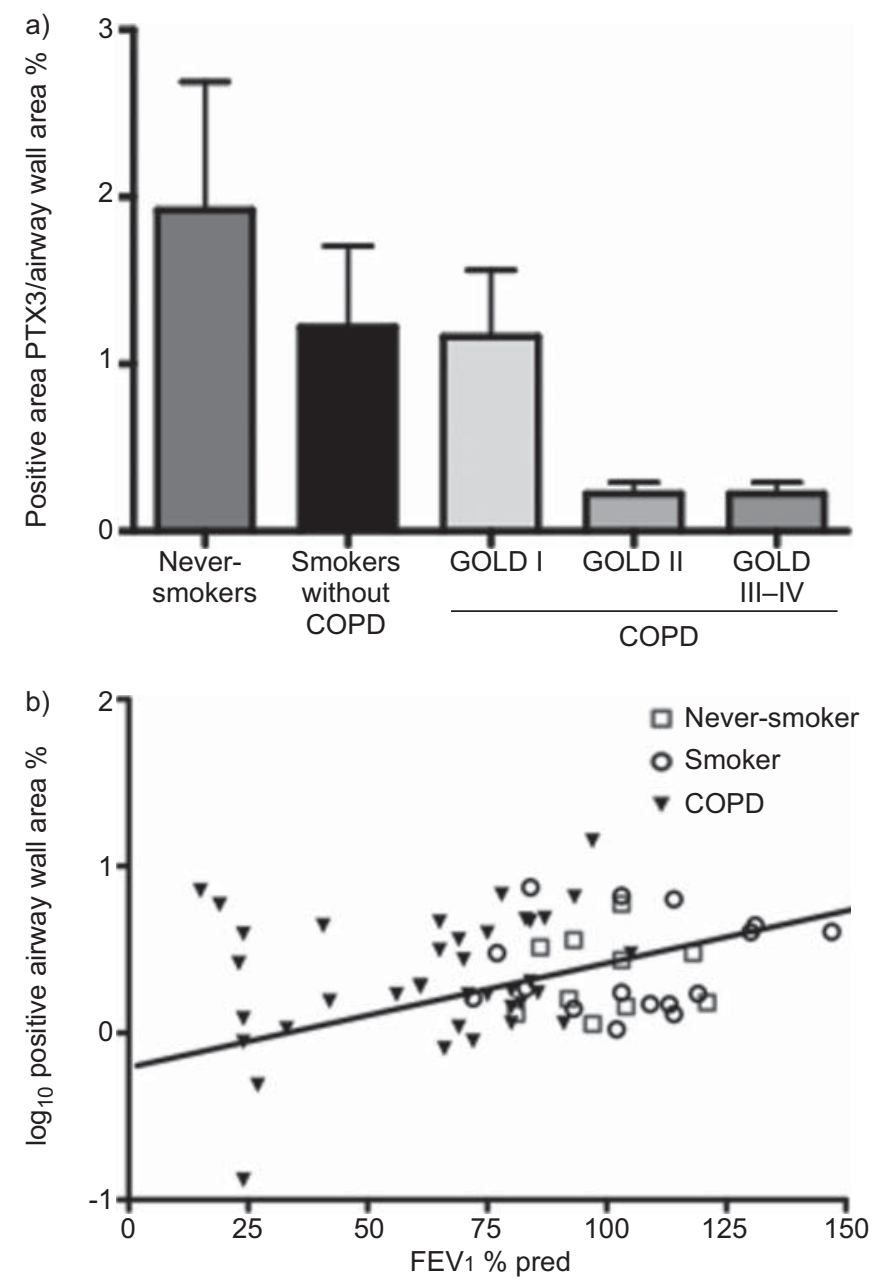

FIGURE 2. Quantification of pentraxin (PTX)3 expression in small airways. Lung tissue sections were stained for PTX3. a) Percentage of the area of the total airway wall that stained positively. Results are shown per study group (neversmokers without airflow limitation, smokers without airflow limitation and patients with chronic obstructive pulmonary disease (COPD) in different Global Initiative for Chronic Obstructive Lung Disease (GOLD) stages). Data are presented as mean \pm SEM. Kruskal-Wallis test: $p>0.05$. b) Scatter plot of the $\%$ predicted $(\%$ pred) value of the post-bronchodilator forced expiratory volume in $1 \mathrm{~s}$ (FEV1), plotted against the percentage of the area of the airway wall that stained positively for PTX3. Each data point represents the mean expression of PTX3 in the small airways of one subject. —: $r=0.35 . p=0.004$.

arteries or alveolar walls. Although this finding may be surprising at first, there is a plausible explanation: the study groups are defined by the GOLD classification, which uses FEV1 to categorise the subjects. However, lower alveolar and vascular PTX3 expression is associated with reduced KCO, which is a marker for emphysema. As COPD is a heterogeneous disease, patients with severe COPD (i.e. severe airflow limitation) can have various levels of emphysema (with, for instance, a limited degree of emphysema and, therefore, relatively normal levels of KCO and PTX3).

At the mRNA level, PTX3 expression did not correlate with lung function parameters. At first sight, this result seems contradictory to the results of PTX3 protein expression levels. There are, however, several possible explanations. First,

\begin{tabular}{|c|c|c|}
\hline TABLE 2 & \multicolumn{2}{|c|}{$\begin{array}{l}\text { Univariate analysis of immunohistochemical } \\
\text { staining of pentraxin (PTX) } 3 \text { in small airways }\end{array}$} \\
\hline \multicolumn{2}{|c|}{ Independent variable } & $\begin{array}{l}\log _{10} \text { PTX3 (area \%) } \\
\text { correlation coefficient } r\end{array}$ \\
\hline \multicolumn{2}{|l|}{ Age } & 0.17 \\
\hline \multicolumn{2}{|l|}{ Sex } & 0.20 \\
\hline \multicolumn{2}{|c|}{ Smoking exposure pack-yrs } & -0.17 \\
\hline \multicolumn{2}{|c|}{ Current smoking status } & -0.27 \\
\hline \multicolumn{2}{|c|}{ Time quit smoking yrs } & 0.13 \\
\hline \multicolumn{2}{|c|}{ Post-bronchodilator FEV 1 L } & 0.20 \\
\hline \multicolumn{2}{|c|}{ Post-bronchodilator FEV $1 \%$ pred } & $0.35^{\star \star}$ \\
\hline \multicolumn{2}{|c|}{$\mathrm{FEV}_{1} / \mathrm{FVC} \%$} & $0.27^{\star}$ \\
\hline \multicolumn{2}{|c|}{ ICS treatment } & -0.22 \\
\hline \multicolumn{2}{|c|}{ OCS treatment } & 0.01 \\
\hline \multicolumn{2}{|c|}{ Statin treatment } & -0.04 \\
\hline \multicolumn{2}{|c|}{ RAAS blocker ${ }^{\#}$ treatment } & 0.01 \\
\hline \multicolumn{2}{|c|}{ Pre-operative CRP level } & -0.01 \\
\hline \multicolumn{2}{|c|}{ Pre-operative white blood cell count } & -0.23 \\
\hline
\end{tabular}

FEV1: forced expiratory volume in $1 \mathrm{~s}$; \% pred: \% predicted; FVC: forced vital capacity; ICS: inhaled corticosteroid; OCS: oral corticosteroid; RAAS: renin/ angiotensin/aldosterone system; CRP: C-reactive protein. ${ }^{*}$ : angiotensinconverting enzyme inhibitors and sartans. *: $p<0.05$ by Pearson correlation coefficient calculation; **: $p<0.01$ by Pearson correlation coefficient calculation.

mRNA levels were measured in total lung tissue samples, which comprise different structures present in the lung, such as blood vessels, airways, the alveolar compartment and lymphoid follicles. This overall mixture of cell types could mask the specific decline in PTX3 levels in the small airway wall and in the alveolar compartment. Secondly, altered posttranscriptional regulation in patients with COPD could result in a decrease of PTX3 at protein level, but not at mRNA level.

When assessing PTX3 levels in induced sputum of patients with COPD and subjects without airflow limitation, we found no association with FEV1. PIZZICHINI et al. [31] also detected PTX3 in induced sputum. In contrast to our findings, PIZZICHINI et al. [31] found an increased level of PTX3 in the sputum of patients with COPD compared with controls. The levels reported by PIZZICHINI et al. [31] are lower than the PTX3 concentrations in our study. The reason for these differences could be multifactorial, including differences in patient selection and sputum processing. In our study, the concentration of PTX3 in induced sputum correlated with the percentage of neutrophils in the sputum. This evidence suggests that the level of PTX3 in sputum is largely dependent on the storage of this innate molecule in neutrophilic granules. This finding is compatible with previous reports on storage of PTX3 in neutrophils [32].

In the systemic circulation, PTX3 levels were not significantly correlated with disease severity of COPD. This is in contrast with the marked increase of plasma CRP in patients with COPD, confirming previous reports on systemic inflammation in COPD [33]. Importantly, within the group of patients with COPD, a significant positive correlation between plasma PTX3 
TABLE 3 Multivariate analysis by linear regression of quantification of pentraxin (PTX)3 staining in small airways

\begin{tabular}{|c|c|c|c|c|}
\hline Outcome variable & $r^{2}$ & Predictor variable & $\beta$ & $\mathrm{p}$-value \\
\hline \multirow[t]{3}{*}{ PTX3 \% airway wall } & 0.24 & FEV $1 \%$ pred & 0.49 & 0.016 \\
\hline & & Ocs & 0.38 & 0.012 \\
\hline & & Time quit smoking yrs & -0.08 & 0.572 \\
\hline \multirow[t]{2}{*}{ PTX3/length of pBM } & 0.25 & FEV $1 \%$ pred & 0.47 & 0.020 \\
\hline & & Time quit smoking yrs & -0.13 & 0.350 \\
\hline
\end{tabular}

levels and FEV1 was again observed, suggesting a decrease of circulating PTX3 levels in patients with a more advanced stage of COPD.

Taken together, we demonstrated that a decrease in pulmonary interstitial and circulating PTX3 protein levels is associated with increased airway obstruction and emphysema in COPD, when measured under clinically stable conditions. This association between lung function parameters of COPD and expression of PTX3 is by no means evidence of a causal relationship. In fact, hypotheses could be generated in both directions: COPD could induce a depletion of PTX3 (through enhanced proteolytic destruction of PTX3 in COPD) and/or the reduced expression of PTX3 might contribute to the pathogenesis of COPD. Our study provides evidence that the decrease in pulmonary PTX3 is more likely to be the consequence of the process of COPD, as PTX3 protein levels are mainly reduced in more advanced stages of COPD (GOLD II-IV). In addition, the absence of a clear association between the lung function parameters and PTX3 expression at the mRNA level further supports the concept of secondary depletion of PTX3 in COPD. Finally, a recent study by our group showed that PTX3 knockout mice chronically exposed to cigarette smoke have similar pulmonary inflammation and emphysema scores as their wild-type controls, arguing against a primary role of PTX3 deficiency in the pathogenesis of COPD [34].

These findings do not exclude the possibility of a secondary reduction of PTX3 protein levels in the lungs of patients with COPD, significantly contributing to the progression of the disease in humans by different mechanisms, both structural and inflammatory. As mentioned previously, low levels of interstitial PTX3 could lead to reduced hyaluronic acid crosslinking with instability of the extracellular matrix, enhancing the process of emphysema and the ongoing inflammation by release of low molecular weight hyaluronic acid fragments. Indeed, several studies have described alterations in pulmonary extracellular matrix components in COPD [35-37].

Reduced circulating PTX3 levels and reduced interstitial PTX3 could contribute to impaired antimicrobial defence, especially during invasive infections. This impaired innate immune function in patients with COPD is compatible with the increased risk of invasive pulmonary infections with
Aspergillus fumigatus and Pseudomonas aeruginosa in these patients [38-40]. As PTX3 is known to be involved in clearance of apoptotic cells, a reduction of the level of this molecule could lead to accumulation of apoptotic cells in COPD [41, 42], which is a possible risk factor for an autoimmune component in COPD pathogenesis. Finally, as PTX3 has a dampening function on neutrophil influx during inflammation [43], reduced pulmonary PTX3 levels could contribute to disproportionate neutrophilic influx, with damaging consequences for the lung tissue.

From the point of view of extrapulmonary effects in COPD, it appears that plasma PTX3 is not useful as a marker to detect systemic inflammation compared with CRP. Elevated systemic levels of PTX3 have been observed in situations of severe inflammatory responses, such as invasive infections and sepsis [44]. We hypothesise that during COPD exacerbations and invasive pulmonary infections, PTX3 concentrations will increase rapidly, both locally and systemically, due to the swift release of PTX3 stored in neutrophils and the enhanced production by white blood cells that are attracted towards the mucosal area of inflammation. Further studies are needed to investigate this topic.

There are different components that contribute to the strength of this study. First, this study examined the expression of PTX3, both at the mRNA and protein levels, in different large study populations, covering both healthy subjects and patients with the different stages of COPD. Secondly, expression of PTX3 was assessed in different compartments (sputum, small airways, alveoli and plasma), resulting in a broad coverage of PTX3 expression relevant to respiratory disease. Thirdly, the described association of lower PTX3 levels in human lungs with increasing airflow limitation was adjusted for possible confounders, suggesting a true relationship with the disease rather than a bystander effect. Finally, expression of PTX3 in different compartments could be linked to the known physiology of PTX3 production (TNF- $\alpha$ ) and storage (sputum neutrophils).

However, there are several limitations of the study that should be addressed. First, for evaluating the expression of PTX3 in human lung tissue, samples were used from patients who were operated for solitary pulmonary lesions, which might influence 

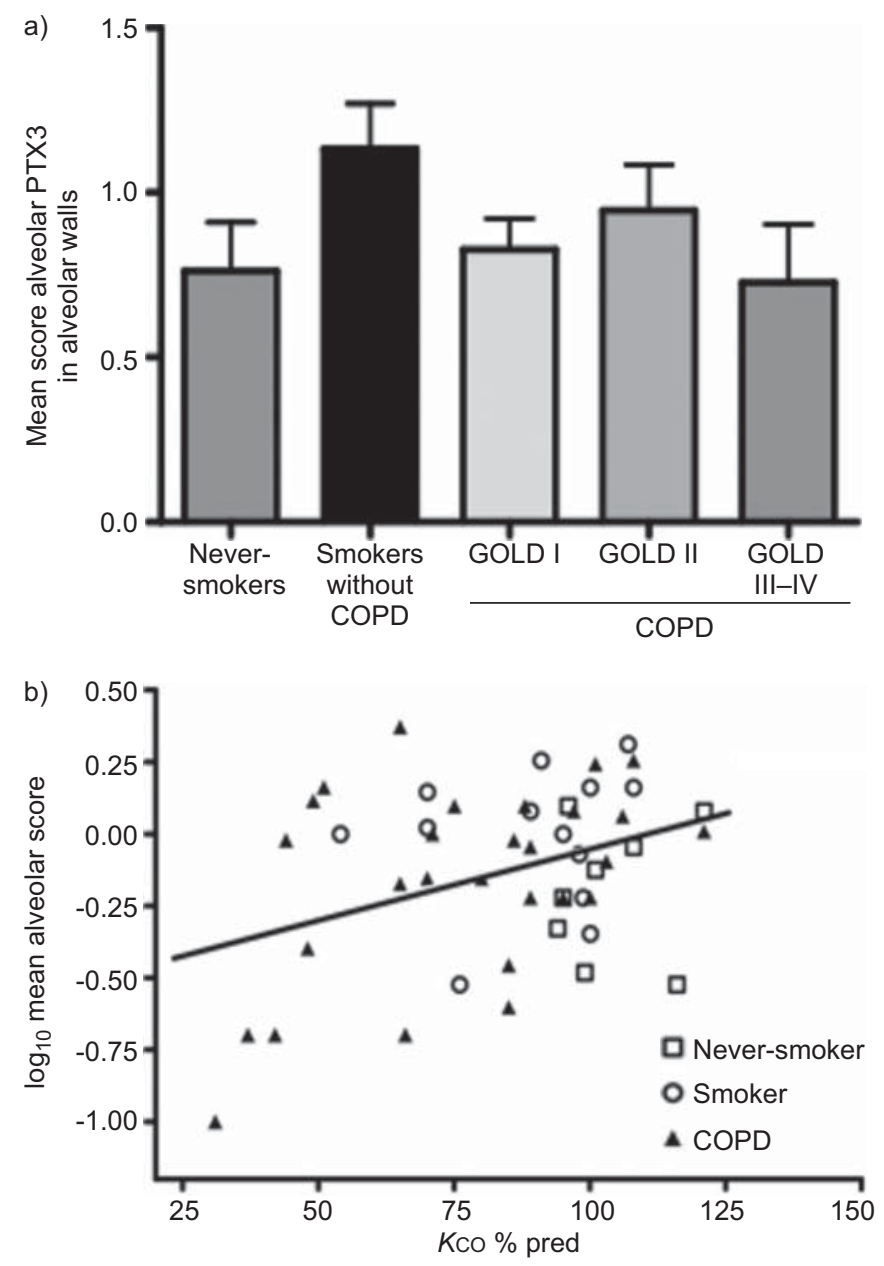

FIGURE 3. Quantification of pentraxin (PTX)3 expression in alveoli. Lung tissue sections were stained for PTX3. a) Mean score of alveolar expression of PTX3 that stained positively per study group (never-smokers without airflow limitation, smokers without airflow limitation and patients with chronic obstructive pulmonary disease (COPD) in different Global Initiative for Chronic Obstructive Lung Disease (GOLD) stages). Data are presented as mean \pm SEM. b) Scatter plot of the transfer coefficient of the lung for carbon monoxide $(\mathrm{KCO})$ plotted against the mean alveolar score for expression of PTX3. \% pred: \% predicted. —: $r=0.28 . p=0.04$.

the expression of acute phase proteins. To minimise the effect of the tumours, great care was taken to obtain tissue samples at a maximal distance from the lesion that were free from retroobstructive pneumonia. Secondly, PTX3 levels were measured in sputum and plasma in separate populations that covered only the mildest stages of COPD, rendering no information on sputum and systemic PTX3 levels in end-stage COPD.

\section{Conclusion}

In this study, we described the localisation of PTX3 expression in the human lung and revealed a positive correlation between pulmonary PTX3 expression and lung function parameters of airflow limitation and emphysema. These data indicate that COPD is accompanied by a marked decrease in PTX3 levels, especially in the interstitial compartment of small airways, which could have important structural and functional inflammatory consequences. Further studies are needed to evaluate
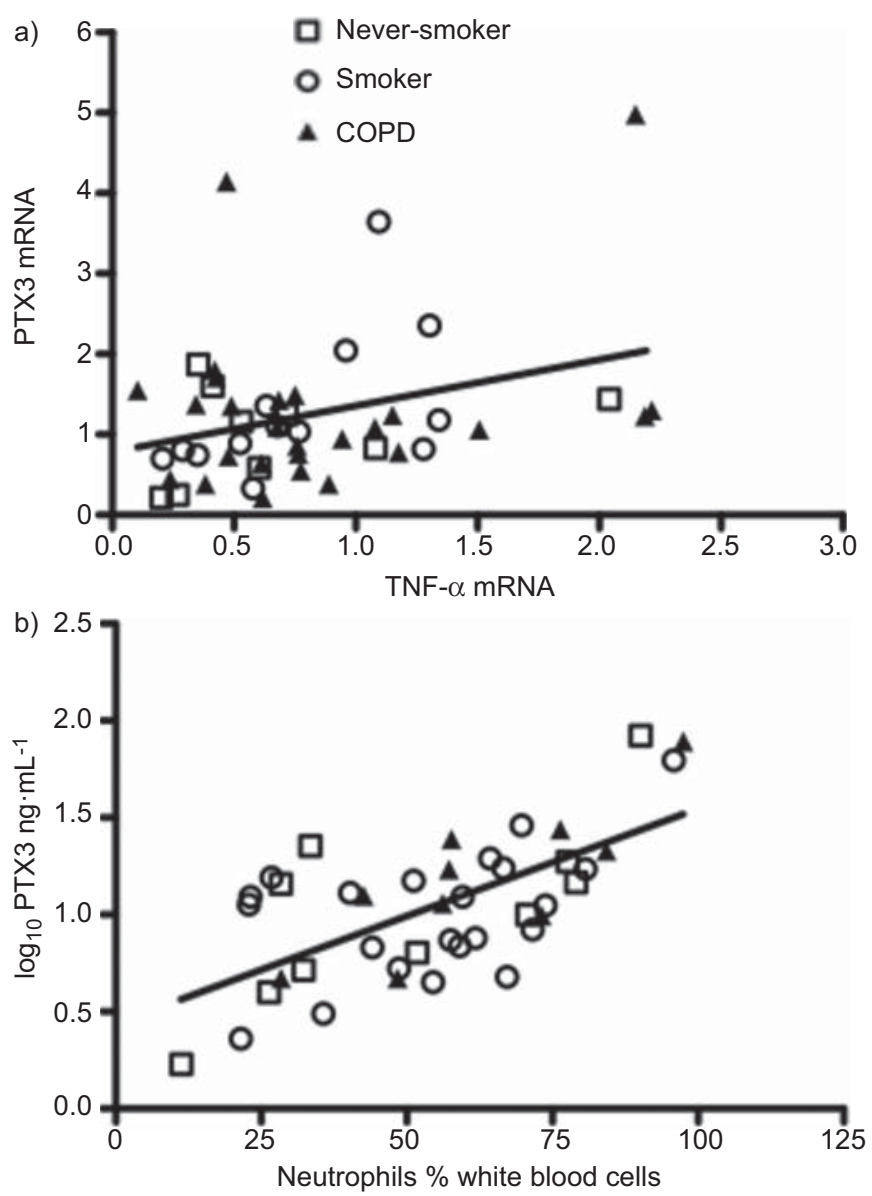

FIGURE 4. Quantification of pentraxin (PTX)3 and tumour necrosis factor (TNF)- $\alpha$ at the mRNA level in human lung tissue. Quantification of PTX3 protein expression in induced sputum supernatant is shown. The expression of PTX3 and TNF- $\alpha$ at the mRNA level was measured by quantitative RT-PCR. a) Scatter plot of the expression of TNF- $\alpha$ and the expression of PTX3, both measured at mRNA level in human lung tissue. $-: r=0.325 . p=0.024$. b) Scatter plot of the concentration of PTX3 in induced sputum versus the percentage of sputum neutrophils. $-: r=0.66$. $\mathrm{p}<0.001$.

the predictive value and the mechanistic roles of PTX3 in COPD.

\section{SUPPORT STATEMENT}

This work was supported by the Fund for Scientific Research in Flanders (FWO Vlaanderen; research projects G.0011.03, G.0195.09N and $\mathrm{G} 0343.01 \mathrm{~N}$ ) and by project grant 01251504 from the Concerted Research Initiative of Ghent University (Ghent, Belgium). G.R. Van Pottelberge is a doctoral research fellow of the Fund for Scientific Research in Flanders. K.R. Bracke is a post-doctoral research fellow of the Fund for Scientific Research in Flanders.

\section{STATEMENT OF INTEREST}

Statements of interest for G.R. Van Pottelberge and G.G. Brusselle can be found at www.erj.ersjournals.com/site/misc/statements.xhtml

\section{ACKNOWLEDGEMENTS}

The authors would like to thank K. De Rijck, A. Neesen, I. De Borle, K. De Saedeleer, E. Castrique, M. Mouton, P. Degryze A. Goethals and S. Verschraeghen (all Dept of Respiratory Medicine, Ghent University Hospital, Ghent, Belgium) for their technical contribution to this work, 
and H. Middendorp, F. Vandewalle, A. Delporte and T. Verstraete (all Dept of Respiratory Medicine, Ghent University Hospital) for their support in data management. The authors would also like to thank G. Verleden (Dept of Respiratory Medicine, University Hospital Gasthuisberg, Catholic University of Leuven, Leuven, Belgium) for providing the lung tissue samples of patients with COPD GOLD stage IV. The authors would like to thank M. Praet (Dept of Pathology, Ghent University Hospital) for providing the samples for positive control immunohistochemical staining.

\section{REFERENCES}

1 Halpin DM, Miravitlles M. Chronic obstructive pulmonary disease: the disease and its burden to society. Proc Am Thorac Soc 2006; 3: 619-623.

2 Lopez AD, Mathers CD. Measuring the global burden of disease and epidemiological transitions: 2002-2030. Ann Trop Med Parasitol 2006; 100: 481-499.

3 Rabe KF, Hurd S, Anzueto A, et al. Global Strategy for the Diagnosis, Management, and Prevention of Chronic Obstructive Pulmonary Disease: GOLD Executive Summary. Am J Respir Crit Care Med 2007; 176: 532-555.

4 Hogg JC, Chu F, Utokaparch S, et al. The nature of small-airway obstruction in chronic obstructive pulmonary disease. $N$ Engl $J$ Med 2004; 350: 2645-2653.

5 Chung KF, Adcock IM. Multifaceted mechanisms in COPD: inflammation, immunity, and tissue repair and destruction. Eur Respir J 2008; 31: 1334-1356.

6 Seemungal T, Harper-Owen R, Bhowmik A, et al. Respiratory viruses, symptoms, and inflammatory markers in acute exacerbations and stable chronic obstructive pulmonary disease. Am J Respir Crit Care Med 2001; 164: 1618-1623.

7 Zhang M, Li Q, Zhang XY, et al. Relevance of lower airway bacterial colonization, airway inflammation, and pulmonary function in the stable stage of chronic obstructive pulmonary disease. Eur J Clin Microbiol Infect Dis 2010; 29: 1487-1493.

8 Tumkaya M, Atis S, Ozge C, et al. Relationship between airway colonization, inflammation and exacerbation frequency in COPD. Respir Med 2007; 101: 729-737.

9 Curtis JL, Freeman CM, Hogg JC. The immunopathogenesis of chronic obstructive pulmonary disease: insights from recent research. Proc Am Thorac Soc 2007; 4: 512-521.

10 Cosio MG, Saetta M, Agusti A. Immunologic aspects of chronic obstructive pulmonary disease. $N$ Engl J Med 2009; 360: 2445-2454.

11 Taraseviciene-Stewart L, Douglas IS, Nana-Sinkam PS, et al. Is alveolar destruction and emphysema in chronic obstructive pulmonary disease an immune disease? Proc Am Thorac Soc 2006; 3: 687-690.

12 Lee SH, Goswami S, Grudo A, et al. Antielastin autoimmunity in tobacco smoking-induced emphysema. Nat Med 2007; 13: 567-569.

13 Lee GW, Lee TH, Vilcek J. TSG-14, a tumor necrosis factor- and IL-1-inducible protein, is a novel member of the pentaxin family of acute phase proteins. J Immunol 1993; 150: 1804-1812.

14 Nauta AJ, de Haij S, Bottazzi B, et al. Human renal epithelial cells produce the long pentraxin PTX3. Kidney Int 2005; 67: 543-553.

15 Doni A, Peri G, Chieppa M, et al. Production of the soluble pattern recognition receptor PTX3 by myeloid, but not plasmacytoid, dendritic cells. Eur J Immunol 2003; 33: 2886-2893.

16 Jaillon S, Peri G, Delneste $Y$, et al. The humoral pattern recognition receptor PTX3 is stored in neutrophil granules and localizes in extracellular traps. J Exp Med 2007; 204: 793-804.

17 Garlanda C, Bottazzi B, Bastone A, et al. Pentraxins at the crossroads between innate immunity, inflammation, matrix deposition and female fertility. Ann Rev Immunol 2005; 23: 337-366.
18 Wisniewski HG, Vilcek J. Cytokine-induced gene expression at the crossroads of innate immunity, inflammation and fertility: TSG-6 and PTX3/TSG-14. Cytokine Growth Factor Rev 2004; 15: 129-146.

19 Muller B, Peri G, Doni A, et al. Circulating levels of the long pentraxin PTX3 correlate with severity of infection in critically ill patients. Crit Care Med 2001; 29: 1404-1407.

20 Norata GD, Garlanda C, Catapano AL. The long pentraxin PTX3: a modulator of the immunoinflammatory response in atherosclerosis and cardiovascular diseases. Trends Cardiovasc Med 2010; 20: $35-40$.

21 Luchetti MM, Piccinini G, Mantovani A, et al. Expression and production of the long pentraxin PTX3 in rheumatoid arthritis (RA). Clin Exp Immunol 2000; 119: 196-202.

22 Ogawa T, Kawano Y, Imamura T, et al. Reciprocal contribution of pentraxin 3 and $\mathrm{C}$-reactive protein to obesity and metabolic syndrome. Obesity (Silver Spring) 2010; 18: 1871-1874.

$23 \mathrm{He}$ X, Han B, Liu M. Long pentraxin 3 in pulmonary infection and acute lung injury. Am J Physiol Lung Cell Mol Physiol 2007; 292: L1039-L1049.

24 Demedts IK, Morel-Montero A, Lebecque S, et al. Elevated MMP12 protein levels in induced sputum from patients with COPD. Thorax 2006; 61: 196-201.

25 Souza DG, Soares AC, Pinho V, et al. Increased mortality and inflammation in tumor necrosis factor-stimulated gene-14 transgenic mice after ischemia and reperfusion injury. Am J Pathol 2002; 160: 1755-1765.

26 Garlanda C, Hirsch E, Bozza S, et al. Non-redundant role of the long pentraxin PTX3 in anti-fungal innate immune response. Nature 2002; 420: 182-186.

27 Han B, Mura M, Andrade CF, et al. TNF $\alpha$-induced long pentraxin PTX3 expression in human lung epithelial cells via JNK. J Immunol 2005; 175: 8303-8311.

28 Okutani D, Han B, Mura M, et al. High-volume ventilation induces pentraxin 3 expression in multiple acute lung injury models in rats. Am J Physiol Lung Cell Mol Physiol 2007; 292: L144-L153.

29 Nebuloni M, Pasqualini F, Zerbi P, et al. PTX3 expression in the heart tissues of patients with myocardial infarction and infectious myocarditis. Cardiovasc Pathol 2011; 20: e27-e35.

30 Nauta AJ, de Haij S, Bottazzi B, et al. Human renal epithelial cells produce the long pentraxin PTX3. Kidney Int 2005; 67: 543-553.

31 Pizzichini MM, Kleveston T, Morato EF, et al. Pentraxin 3 (PTX3): a new marker to study airway inflammation? Am J Respir Crit Care Med 2009; 179: A2532.

32 Jaillon S, Peri G, Delneste Y, et al. The humoral pattern recognition receptor PTX3 is stored in neutrophil granules and localizes in extracellular traps. J Exp Med 2007; 204: 793-804.

33 Sin DD, Man SF. Why are patients with chronic obstructive pulmonary disease at increased risk of cardiovascular diseases? The potential role of systemic inflammation in chronic obstructive pulmonary disease. Circulation 2003; 107: 1514-1519.

34 Pauwels NS, Bracke KR, Maes T, et al. Cigarette smoke induces PTX3 expression in pulmonary veins of mice in an IL-1 dependent manner. Respir Res 2010; 11: 134.

35 Bracke KR, Dentener MA, Papakonstantinou E, et al. Enhanced deposition of low-molecular-weight hyaluronan in lungs of cigarette smoke-exposed mice. Am J Respir Cell Mol Biol 2010; 42: 753-761.

36 Dentener MA, Vernooy JH, Hendriks S, et al. Enhanced levels of hyaluronan in lungs of patients with COPD: relationship with lung function and local inflammation. Thorax 2005; 60: 114-119.

$37 \mathrm{Li} \mathrm{H}$, Cui $\mathrm{D}$, Ma $\mathrm{N}$, et al. The effect of extracellular matrix remodeling on airflow obstruction in a rat model of chronic obstructive pulmonary disease. Zhonghua Jie He He Hu Xi Za Zhi 2002; 25: 403-407.

38 Arancibia F, Bauer TT, Ewig S, et al. Community-acquired pneumonia due to Gram-negative bacteria and Pseudomonas 
aeruginosa: incidence, risk, and prognosis. Arch Intern Med 2002; 162: 1849-1858.

39 Falguera M, Carratala J, Ruiz-Gonzalez A, et al. Risk factors and outcome of community-acquired pneumonia due to Gramnegative bacilli. Respirology 2009; 14: 105-111.

40 Cornillet A, Camus C, Nimubona S, et al. Comparison of epidemiological, clinical, and biological features of invasive aspergillosis in neutropenic and nonneutropenic patients: a 6-year survey. Clin Infect Dis 2006; 43: 577-584.

41 Demedts IK, Demoor T, Bracke KR, et al. Role of apoptosis in the pathogenesis of COPD and pulmonary emphysema. Respir Res 2006; 7: 53.
42 Baruah P, Propato A, Dumitriu IE, et al. The pattern recognition receptor PTX3 is recruited at the synapse between dying and dendritic cells, and edits the cross-presentation of self, viral, and tumor antigens. Blood 2006; 107: 151-158.

43 Deban L, Russo RC, Sironi M, et al. Regulation of leukocyte recruitment by the long pentraxin PTX3. Nat Immunol 2010; 11: 328-334.

44 Al-Ramadi BK, Ellis M, Pasqualini F, et al. Selective induction of pentraxin 3, a soluble innate immune pattern recognition receptor, in infectious episodes in patients with haematological malignancy. Clin Immunol 2004; 112: 221-224. 